\title{
Isolation of newer probiotic microorganisms from unconventional sources
}

\section{Manpreet Kaur}

Department of Microbiology, Shaheed Udham Singh College of Research and Technology, Mohali -140306 (Punjab), India

\section{Gaganpreet Kaur}

Department of Microbial Biotechnology, Panjab University, Chandigarh, India Amita Sharma*

Department of Agriculture, Shaheed Udham Singh College of Research and Technology, Mohali -140306 (Punjab), India

${ }^{*}$ Corresponding author. E-mail: amita.sharma22@gmail.com

\begin{abstract}
Probiotics are live microbes in the form of dried or fermented cells that are highly beneficial for human health. The food industry has been revolutionised due to introduction of potential probiotic organisms in a varied formulations derived from mostly dairy products. Isolation of probiotic microbes from unexplored non-dairy sources is gaining attention these days. In the present study, six potential probiotic isolates from non-dairy sources were obtained that are also biocompatible with each other. Out of these, five isolates were gram positive rod shaped and one was gram negative rod shaped. These isolates were able to grow in presence of lysozyme, low $\mathrm{pH}$ and bile salts with good adherence ability. The market for probiotic microorganisms from unconventional products is accelerating to deal with lactose intolerant people. These probiotic attribute studies revealed their potential to be exploited at industrial scale.
\end{abstract}

Keywords: Antimicrobial activity, bile tolerance, fermented cells, hydrophobicity, probiotics

\section{INTRODUCTION}

Probiotics are generally live microbes that can be isolated from dietary supplements, food products and drugs and have a beneficial effect on human health (Sanders et al., 2003; Parvez et al., 2006). For probiotic delivery numerous food products like yoghurt, cheese, cereals, smoothies, juices, fermented and unfermented milks are available in the market. In addition to these sources various dried and packaged microbes in the form of tablets, capsules and also as dietary supplements are available in the market. These commercial health supporting products are either single microorganism or may be combination of more than one microbe. In human adults, bacterial divisions that dominate accounting for more than $90 \%$ microbiota are Bacteriodes and Firmicutes. Other important bacteria include Proteobacteria, Fusobacteria, Acitonobacteria and Verrucomicrobia. According to the 2015 Grand View Research report the global share of probiotics in industries reached near about US \$32.06 billion (Merenstein et al., 2017)

Probiotics are exploited these days to maintain a good, healthy balance of gut bacterial ecosystem.

\section{Article Info}

DOI:10.31018/jans.v10i3.1724

Received: Mar 4, 2018

Revised: June 28, 2018

Accepted: July 5, 2018$$
\text { - }
$$

\section{How to Cite}

Kaur, M. et al. (2018).

Isolation of newer probiotic microorganisms from unconventional sources. Journal of Applied and Natural Science, 10(3): 847 - 852
Microbial imbalance is a change in diversity richness and stability of the gut microbiota. This microbial imbalance or dysbiosis can lead to various intestinal disorders like bowl syndrome, colorectal cancer etc. This can be prevented with the use of probiotics in diet (Vasiljevic et al., 2008). These sources have capability to improve intestinal micro flora of ileum and colon (Ezendam et al., 2006; Collado et al., 2007; Markowiak et al., 2017) and are considered as microbial dietary adjuvant that improve host mucosal and systemic immunity (Colbey et al. 2018). The existence of normal micro-flora in gastrointestinal tract helps to maintain the integrity of the epithelial barrier and also help to develop mucosal immunity (O'Hara et al., 2007). Any disturbance in normal micro-flora may lead to pathogenesis of numerous disorders such as inflammatory bowel disorder, autoimmune diseases, colon cancer, gastric ulcers, cardiovascular diseases and obesity (Bien et al., 2013). Therefore, it becomes very important to restore the normal micro-flora and one way to regain it is through the use of prebiotics and probiotics. Prebiotics are the fermented ingredients that cause changes in the composition or activity of gut microflora in a specific way. These are non-digestible by the host 
but provide health benefits via positive impact on the native gastrointestinal microbes. Mostly prebiotics are provided to the host as ingredients of different food like cereals, biscuits, chocolates, bread spreads and dairy products.

There are numerous barriers in the human body with antimicrobial activity that prevent the growth of external microorganisms. For example lysozyme, present in saliva exhibit antibacterial activity against Gram positive bacteria and breaks the cell wall of bacteria. Other barriers are high acidic conditions and high bile salt concentration, In order to provide health benefits probiotic strains should have capability to survive these conditions (Munoz-Quezada et al., 2013). The probiotic bacteria must be hydrophobic enough to adhere well to the ileum and colon wall. Lactobacillus and Bifidobacterium are the most commonly used microorganisms as probiotics which are found in large numbers in gut of healthy humans and have GRAS (Generally Regarded As Safe) status (Shokryazdan et al., 2014).

Now days, research has been focused for isolating the probiotic strains from non-dairy sources like soil, fruit juices, grains etc (Sornplang et al., 2016). Keeping above points in mind the work was designed to screen probiotic bacteria from unconventional food sources to utilize them commercially specifically for lactose-intolerant people.

\section{MATERIALS AND METHODS}

Isolation of probiotic strains from unconventional sources: The unconventional samples like brockley (BR), carrot (CR), cucumber (CC), whitegram (WG), moong (MG), shakkar (SH), green beans (GB), honey (HN), papaya (PP), wheat $(\mathrm{WH})$ were collected. These samples were serially diluted ten-folds and dilutions were spread on De Man, Rogosa and Sharpe (MRS) agar medium (proteose peptone $10 \mathrm{gm}$, beef extract $10 \mathrm{gm}$, yeast extract $5 \mathrm{gm}$, dextrose $20 \mathrm{gm}$, polysorbate (80) $1 \mathrm{gm}$, ammonium citrate $2 \mathrm{gm}$, sodium acetate $5 \mathrm{gm}$, magnesium sulphate $0.1 \mathrm{gm}$, manganese sulphate $0.050 \mathrm{gm}$, dipotassium phosphate $2 \mathrm{gm}$, agar $12 \mathrm{gm}$, final $\mathrm{pH}$ of 6.5 )

The plates were incubated at $37^{\circ} \mathrm{C}$ for $24 \mathrm{~h}$ $48 \mathrm{~h}$. The plates were observed for two days to analyze any appearance if colonies on each plate. The isolates were further purified by streak method on MRS agar plates followed by incubation at $37^{\circ} \mathrm{C}$ for $24 \mathrm{~h}$. The purified isolates were maintained on MRS agar slants at $4^{\circ} \mathrm{C}$ (Prawan et al., 2017).

Identification of different probiotic bacteria: Gram staining and biochemical test (citrate test, urease test, indole test, MR (Methyl Red) test, VP (Voges Proskauer) test, catalase test, TSI (triple sugar iron) test were performed for characterization of selected isolates.

Citrate test (Simmons, 1926): Simmons citrate media (24.28 gm) was dissolved in water and volume made up to $1 \mathrm{~L}$ and boiled. It was dispensed in sugar tubes and autoclaved at $15 \mathrm{psi}$ for 15 minutes. The media was allowed to settle as slants. The tubes were streaked using a loop with $24 \mathrm{~h}$ old culture. The tubes were incubated at $37^{\circ}$ $\mathrm{C}$ for $24 \mathrm{~h}$ and observed for the colour change.

Urease test (Christensen, 1946): Peptone and agar was dissolved in water and volume make up to $1 \mathrm{~L}$. This was autoclaved at $15 \mathrm{psi}$ for 15 minutes; to the molten media added urea and phenol red mixed to it. Dispensed in sugar tubes and allowed to settle media as slants. The tubes were inoculated with $24 \mathrm{~h}$ old culture by streaking on the slant. The tubes were incubated at $37^{\circ} \mathrm{C}$ for $24 \mathrm{~h}$ and change in colour was noted.

Indole test (Isenberg et al., 1958): Prepared trypton broth was autoclaved and culture was inoculated in trypton broth in a test tube after that test tube was incubated at $37^{\circ} \mathrm{C}$ for $24 \mathrm{~h} .1 \mathrm{ml}$ of kovac's reagent was added in inoculated test tube. The test tube was shaken well and allowed to stand to permit the reagent to come on the top of test tube. Appearance of red ring or red layer was observed.

MR test: Prepared MR broth was added in test tube and subjected to be autoclaved. The loopful culture was inoculated in a test tube. The tubes were incubated at $37^{\circ} \mathrm{C}$ for $24 \mathrm{~h}$. 5 drops of methyl red solution was added in inoculated test tube and change in colour from yellow to red indicates positive test.

VP test (Voges et al., 1898): MRVP broth was prepared and poured in test tubes and autoclaved. The culture was inoculated in test tube containing broth. The test tubes were incubated at $37^{\circ} \mathrm{C}$ for 24h. 12 drops of V-P-1 and 2-3 drops of V-P-2 reagent were added in inoculated tubes and kept for some time. The change in colour from yellow to red was taken as positive.

Catalase test (Taylor et al., 1972): The clean glass slides were used. A drop of hydrogen peroxide was placed on it. Using a loop/ toothpick a culture was picked from a $24 \mathrm{~h}$ old culture and mixed with the drop of $\mathrm{H}_{2} \mathrm{O}_{2}$ and noted for the appearance of bubbles.

TSI test: TSI agar was inoculated by first stabbing into the center of the medium up to the tube bottom followed by streaking on the agar slant surface with $24 \mathrm{~h}$ grown culture. The tubes were incubated at $37^{\circ} \mathrm{C}$ for $18-24 \mathrm{~h}$.

Probiotic attributes: The selected 10 isolates (BR3, CR1, CC4, CH1, MG6, SH1, GB4, HN1, $\mathrm{PP} 1, \mathrm{WH} 2)$ were further studied for various probiotic attributes like low pH tolerance, bile tolerance, lysozyme tolerance, bacterial surface hydrophobicity and anti-microbial activity (Trikha et al., 2015). i.) Lysozyme tolerance: Ten selected isolates were checked for lysozyme tolerance by inoculating them in MRS broth supplemented with $1 \%$ 
lysozyme (Saran et al., 2012). The inoculated media was incubated for $4 \mathrm{~h}$ at $37^{\circ} \mathrm{C}$ under static condition. The control samples inoculated with each isolate (no lysozyme) were incubated at same conditions. After each hour, the small amount of sample was withdrawn for analysis of growth using spectrophotometer (UVI VIS Spectrophotometer UV $3000+$, Lab, India) at 600nm.

ii). Low pH tolerance: The probiotic microorganisms should be acid tolerant therefore the tolerance of isolates to acidic conditions was checked by growing them in MRS broth $(\mathrm{pH} 2.5)$. The medium was inoculated with selected ten isolates (grown for $16 \mathrm{~h}$ ) and incubated for $4 \mathrm{~h}$ at $37^{\circ} \mathrm{C}$ under static condition. After each hour, the small amount of sample was withdrawn for analysis of growth using spectrophotometer (UVI VIS Spectrophotometer UV 3000+, Lab, India) at 600nm.

iii). Bile tolerance: The tolerance of microorganisms was checked by growing them in MRS broth supplemented with $0.5 \%$ ox-bile .The inoculated medium was incubated for $4 \mathrm{~h}$ at $37^{\circ} \mathrm{C}$ under static condition. After each hour, the small amount of sample was withdrawn for analysis of growth using spectrophotometer (UVI VIS Spectrophotometer UV 3000+, Lab, India) at 600nm.

iv). Bacterial surface hydrophobicity: Hydrophobic interactions were tested using xylenewater system (BATH- bacterial adherence to hydrocarbons). Hydrophobicity tests the ability of microorganism to adhere and colonize ileum/colon to confer health benefits. In order to test hydrophobicity the isolates were grown in MRS broth for $16 \mathrm{~h}-18 \mathrm{~h}$ at $37^{\circ} \mathrm{C}$ followed by centrifugation at $10,000 \mathrm{rpm}$ for 10 minutes. The pellet obtained after centrifugation was washed and suspended in PBS (phosphate buffer saline) buffer. The absorbance of the suspension was adjusted to 1.0 at $560 \mathrm{~nm}$ and $600 \mu \mathrm{l}$ of xylene was added.

V). Antimicrobial activity: The agar well diffusion method was used for antimicrobial activity of probiotics against selected strains Escherichia coli (MTCC 2961), Staphylococcus aureus (MTCC 3160). The isolates were grown in MRS broth for $16 \mathrm{~h}-18 \mathrm{~h}$ at $37^{\circ} \mathrm{C}$ followed by centrifugation at

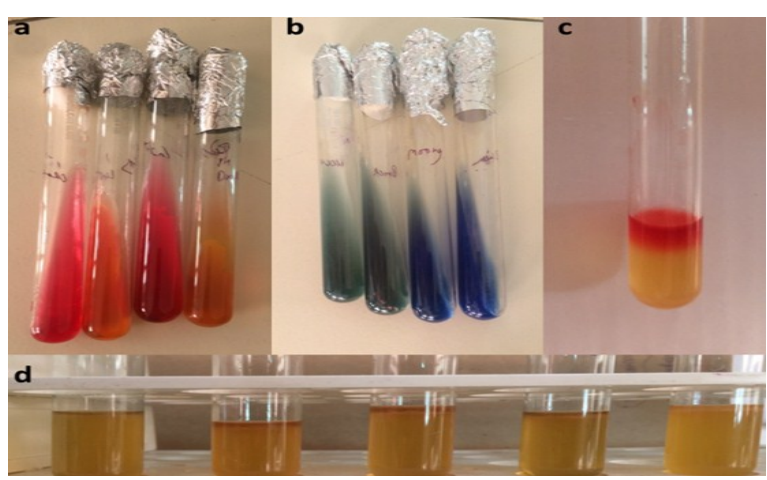

Fig.1. Results of Biochemical tests (a) TSI test (b) Citrate test (c) MR (methyl red) test (d) VP test.
$10,000 \mathrm{rpm}$ for 10 minutes. The supernatant was collected after centrifugation. The MHA plates were spread with test pathogens and $100 \mu$ l of supernatant was added to the well. The plates were incubated for $24 \mathrm{~h}$ at $37^{\circ} \mathrm{C}$ (Valgas et al., 2007).

Biocompatibility test: The biocompatibility of selected isolates was tested to ensure that they don't cause inhibition of each other. The test was performed by perpendicular streaking of isolate to each other separated by $0.5 \mathrm{~mm}$ distance on on MRS agar plates. After incubation at $37^{\circ} \mathrm{C}$ for $24 \mathrm{~h}$ the plates were checked for the growth of isolates (Trikha et al., 2015).

\section{RESULTS}

In the present study, different non-dairy sources were collected for isolation of probiotic microorganisms. Each sample was processed by serial dilution method and different number of isolates was obtained. Table 1 shows the non-dairy sources and number of isolates obtained from each sample after incubation on agar plates. Total of 34 isolates were obtained from all these samples. Out of which, 10 isolates were selected for further studies on basis of colony morphology and gram staining. The selected isolates were subjected to gram staining and biochemical characterization. Table 2 summarizes the gram character of the selected isolates from different unconventional sources. Out of 10 isolates, 8 were gram positive rods and 2 were gram negative rods. The figure 1 and table 3 summarized the biochemical test of the selected isolates.

Probiotic attributes: The selected 10 isolates were tested for probiotic characteristics such as to lysozyme tolerance, acid tolerance, bile tolerance and cell surface hydrophobicity. In the lysozyme tolerance test, all the isolates showed nearly equal significantly good growth in the presence of lysozyme suggesting the ability of these isolates to tolerate lysozyme. These isolates were further analyzed for growth in low $\mathrm{pH}$ conditions. Out of these isolates, CC4 and PP1 showed best growth at acidic conditions. The isolates $\mathrm{BR} 3, \mathrm{CC} 4, \mathrm{CH} 1$ and PP1 showed maximum tolerance to bile. Hydro-

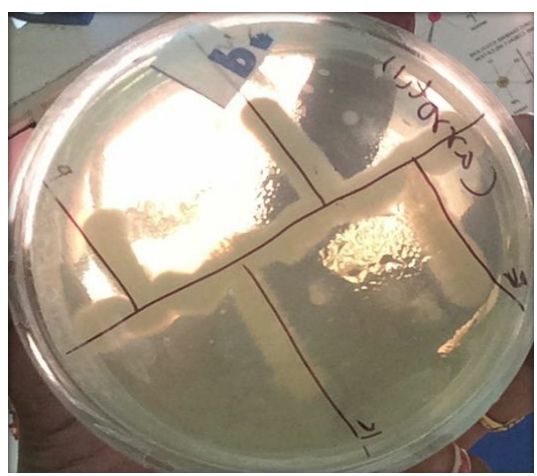

Fig. 2. Biocompatibility test of isolates. 
Table 1. Different unconventional sources of probiotics and number of isolates from each source.

\begin{tabular}{|c|c|c|}
\hline S.N. & Unconventional sources of probiotics & No. of isolates \\
\hline 1 & Brockley (BR) & 4 (BR1,BR2,BR3,BR4) \\
\hline 2 & Carrot (CR) & 2 (CR1,CR2) \\
\hline 3 & Cucumber (CC) & $5(\mathrm{CC} 1, \mathrm{CC} 2, \mathrm{CC} 3, \mathrm{CC} 4, \mathrm{CC} 5)$ \\
\hline 4 & Channe $(\mathrm{CH})$ & $4(\mathrm{CH} 1, \mathrm{CH} 2, \mathrm{CH} 3, \mathrm{CH} 4)$ \\
\hline 5 & Moong (MG) & 7 (MG1,MG2,MG3,MG4,MG5,MG6,MG7) \\
\hline 6 & Shakkar (SH) & $3(\mathrm{SH} 1, \mathrm{SH} 2, \mathrm{SH} 3)$ \\
\hline 7 & Green beans (GB) & 4 (GB1,GB2,GB3,GB4) \\
\hline 8 & Honey (HN) & 1 (HN1) \\
\hline 9 & Papaya (PP) & 2 (PP1,PP2) \\
\hline 10 & Wheat (WH) & $2(\mathrm{WH} 1, \mathrm{WH} 2)$ \\
\hline
\end{tabular}

Table 2. Gram character of selected isolates.

\begin{tabular}{lll}
\hline S.N. & Isolate No. & Gram Character \\
\hline 1 & BR3 & Gram positive rod shaped \\
2 & CR1 & Gram positive rod shaped \\
3 & CC4 & Gram positive rod shaped \\
4 & CH1 & Gram negative rod shaped \\
5 & MG6 & Gram positive rod shaped \\
6 & SH1 & Gram negative rod shaped \\
7 & GB4 & Gram positive rod shaped \\
8 & HN1 & Gram positive rod shaped \\
9 & PP1 & Gram positive rod shaped \\
10 & WH2 & Gram positive rod shaped \\
\hline
\end{tabular}

phobicity assay was performed to analyse the adherence ability of each isolate. Among the 10 isolates, 6 isolates (BR3, CC4, CH1, MG6, GB4 and PP1) showed good hydrophobicity. The isolates were further selected for antimicrobial activity against Escherichia coli, Staphylococcus aureus but none of them showed the antimicrobial nature. Biocompatibility test was performed to test that consortium of selected probiotic isolates can be prepared further (figure 2). All 6 isolates were biocompatible with each other because no growth inhibition of isolates was observed.

The isolates were further selected for antimicrobial activity against Escherichia coli, Staphylococcus aureus. All the selected 10 isolates showed the complete tolerance to lysozyme, acid and bile. Out of 10 isolates (BR3, CR1, CC4, CH1, MG6, $\mathrm{SH} 1, \mathrm{~GB} 4, \mathrm{HN} 1, \mathrm{PP} 1, \mathrm{WH} 2), 6$ isolates showed significant hydrophobicity as compared to others. The selected 6 isolates (BR3, CC4, CH1, MG6, GB4 and PP1) do not possess antimicrobial activity against test pathogens Escherichia coli and Staphylococcus aureus. Biocompatibility test was performed to test that consortium of selected pro- biotic isolates can be prepared further (figure 2). All 6 isolates were biocompatible with each other.

\section{DISCUSSION}

Probiotics are defined as "live microorganisms which when administered in adequate amounts confer a health benefit on the host" (by FAO and WHO in 2001). They have gained immense attention owing to their beneficial effects on human health. They are considered a healthy alternative to treat and prevent numerous diseases in humans like bowl syndrome, gastritis and several urogenital disorders (Vasile et al., 2011). Antibiotic associated studies have suggested that lactobacilli given at high concentration were potent enough to kill HIV-1 thus revealing the possible role of probiotics in preventing transmission of diseases like STD's and AIDS. The probiotic agents may provide prototype antimicrobial substances that will be useful for pharmaceutical companies in the development of new antibiotics (Vasile et al., 2011).

Bacterial isolates were identified through different biochemical tests such as citrate, catalase, indole, urease, MRVP and triple sugar test (TSI) etc. The selected 10 isolates were further studied for their probiotic attributes such as acid, bile and lysozyme tolerance, cellular hydrophobicity and antimicrobial activity.

The probiotic organisms should have the ability to survive in the acidic environment of human stomach. All the selected isolates showed tolerance to acidic conditions ( $\mathrm{pH}$ 2.5). Tokat et al., 2015 studied the tolerance to acid by numerous lactic acid bacteria and reported that highest tolerance (85\%) was showed by Lactobacillus plantarum MF303.

Table 3. Biochemical characterization of selected isolates.

\begin{tabular}{|c|c|c|c|c|c|c|c|c|}
\hline S.N. & Isolate No. & Catalase & Citrate & Indole & MR & TSI & Urease & VP \\
\hline 1 & BR3 & - & + & - & + & + & - & - \\
\hline 2 & CR1 & - & + & - & + & + & - & - \\
\hline 3 & CC4 & - & + & - & + & + & - & - \\
\hline 4 & $\mathrm{CH} 1$ & + & - & - & + & + & - & + \\
\hline 5 & MG6 & + & - & - & + & + & - & + \\
\hline 6 & $\mathrm{SH} 1$ & + & - & - & + & + & - & - \\
\hline 7 & GB4 & + & + & - & + & + & - & - \\
\hline 8 & HN1 & - & + & - & + & + & - & - \\
\hline 9 & PP1 & - & + & - & + & + & - & - \\
\hline 10 & WH2 & - & + & - & + & - & - & - \\
\hline
\end{tabular}


Survival in the presence of bile salts is also crucial to qualify as a potential probiotic organism (Das et al., 2014). All the selected isolates showed tolerance to $0.5 \%$ ox-bile. Resistance to lysozyme is an important probiotic attribute and therefore the isolates were tested for lysozyme tolerance. All the isolates showed tolerance to lysozyme. Cellular hydrophobicity determines the adherence of the bacterial (probiotic) cells to the intestinal wall. The hydrophobicity studies were carried out for the selected isolates and six isolates (BR3, CC4, $\mathrm{CH} 1, \mathrm{MG} 6, \mathrm{~GB} 4$ and PP1) showed significant hydrophobicity. Biocompatibility tests have revealed that all the isolates were compatible with each other.

\section{Conclusion}

Probiotic microorganisms have been recognised as a healthy alternative to treat numerous diseases in both humans and animals. The use of probiotics is accelerating as this is a considerable approach to decrease drug resistance cases in both humans and animals that occurs due to random and overuse of antibiotics. In the present study total 34 isolates were obtained from unconventional sources. Out of which 10 were selected for studying attributes to qualify as potential probiotic. Six isolates (BR3, CC4, CH1, MG6, GB4 and PP1) showed good probiotic characteristic to explore at commercial level. These isolates were found to be biocompatible so can be used to prepare a healthy cocktail. Further studies dealing with molecular characterization and in vivo studies for evaluation of their effect on digestive and immune system will make them more industry relevant.

\section{ACKNOWLEDGEMENTS}

We acknowledge the Department of Microbiology, Shaheed Udham Singh College of Research and Technology, Tangori for providing all the necessary facilities to carry out this research work.

\section{REFERENCES}

1. Bien, J. Palagani, V. and Bozko, P. (2013). The intestinal microbiota dysbiosis and Clostridium difficile infection: Is there a relationship with inflammatory bowel disease. Therap. Adv. Gastroenterol. 6 (1):53-68.

2. Christensen, W. B. (1946). Urea decomposition as a means of differentiating Proteus and paracolon cultures from each other and from Salmonella and Shigella types. J. Bacteriol. 52: 461-466.

3. Colbey, C. Cox, A.J. Pyne, D.B. Zhang, P. Cripps, A.W. and West, N.P. (2018). Upper respiratory symptoms, gut health and mucosal immunity in athletes. Sports Med. 48: 65-77.

4. Collado, M.C. Meriluoto, J. and Salminen, S. (2007). Role of commercial probiotic strains against human pathogen adhesion to intestinal mucus. Lett. Appl. Microbiol. 45: 454-460.

5. Das, D. and Goyal, A. (2014). Characterization of a non-cytotoxic bacteriocin from probiotic Lactobacillus plantarum DM5 with potential as a food preservative. Food Funct. 5: 2453-2462.

6. Ezendam, J. and Loveren, H. (2006). Probiotics: Immunomodulation and evaluation of safety and efficacy. Nutr. Rev. 64:1-14.

7. Isenberg H. D. and Sundheim, L. H. (1958) Indole reactions in bacteria. J. Bacteriol. 75, 682-690.

8. Markowiak, P. and Slizewska, K. (2017). Effects of Probiotics, Prebiotics, and Synbiotics on Human Health. Nutrients 9:1021.

9. Merenstein, D. and Salminen, S. (2017). Probiotics and prebiotics.

10.Munoz-Quezada, S. Chenoll, E. Vieites, J.M., Genoves, S. Maldonado, J. Bermudez-Brito, M. GomezLlorente, C. Matencio, E. Bernal, M.J. Romero, F. Suarez, A. Ramon, D. and Gil, A. (2013). Isolation, identification and characterization of three novel probiotic strains (Lactobacillus paracasei CNCM I-4034, Bifidobacterium breve CNCM I-4035 and Lactobacillus rhamnosus CNCM I-4036) from the faeces of exclusively breast-fed Infants. Br. J. Nutr. 109:S51S62.

11.O'Hara, A.M. and Shanahan, F. (2007). Gut microbiota: Mining for therapeutic potential. Clin. Gastroenterol. Hepatol. 5:274-284.

12.Parvez, S. Malik, K.A. Kang, K.A. and Kim, H.Y. (2006). Probiotics and their fermented food products are beneficial for health: Review. J. Appl. Microbiol. 100:1171-1185.

13.Prawan, K. and Bhima, B. (2017). Isolation and characterization of lactic acid bacteria for probiotic application from plant sources. Int. J. Adv. Res. 5(4): 869876.

14.Sanders, M.E. Morelli, L. and Tompkins, T.A. (2003). Spore formers as human probiotics: Bacillus, Sporolactobacillus and Brevibacillus. Compr. Rev. Food Sci. Food Safety. 2:101-110.

15.Saran, S. Bisht, M.S. Singh, K. and Teotia, U.S. (2012). Analyzing probiotic attributes to assess comparatively two isolates of Lactobacillus acidophilus in prebiotics, honey and inulin. DHR IJBLS. 2: 26-34.

16.Shokryazdan, P. Sieo, C.C. Kalavathy, R. Liang, J.B. Alitheen, N.B. Jahromi, M.F. and Ho, Y.W. (2014). Probiotic potential of Lactobacillus Strains with antimicrobial activity against some human pathogenic strains. BioMed Res. Int. Volume 2014: Article ID 927268, 16 pages.

17.Simmons, J.S. (1926). A culture media for differentiating organisms of typhoid colon aerogenes groups and for isolation of certain fungi. J. Infect. Dis. 39:209.

18.Sornplang, P. and Piyadeatsoontorn, S. (2016). Probiotic isolates from unconventional sources: A Review. J. Ani. Sci. Technol. 58:26.

19.Taylor, W. I. and Achanzar, D. (1972). Catalase test as an aid to the identification of Enterobacteriaceae. Appl. Microbiol. 24: 58-61.

20.Tokat, M. Gulgor, G. Elmac, S.B. Isleyen, N.A. and Ozcelik, F. (2015). In Vitro properties of potential probiotic indigenous lactic acid bacteria originating from traditional pickles. BioMed Res. Int. Volume 2015: Article ID 315819, 8 pages.

21.Trikha, R. Sharma, A. Guglani, V. Rishi, P. and Tewari, R. (2015). Isolation of probiotic Lactobacilli from human infants stool samples exhibiting antimi- 
Kaur, M. et al. / J. Appl. \& Nat. Sci. 10 (3): 847 - 852 (2018)

crobial activity against pathogenic microorganisms. J. Pure Appl. Microbiol. 9(1):377-390.

22.Valgas, C. De-Souza, S.M. Smania, E.F. and Smania, Jr A. (2007). Screening methods to determine antibacterial activity of natural products. Braz. J. Microbiol. 38:369-380.

23. Vasile, N. Ghindea, R. and Vassu, T. (2011). Probiotics-An alternative treatment for various diseases.
Roum. Arch. Microbiol. Immunol. 70:54-59.

24.Vasiljevic, T. and Shah, N.P. (2008). Review: Probiotics-from metchnikoff to bioactives. Int. Dairy $\mathrm{J}$. 18:714-728.

25.Voges, D. and Proskauer, B. (1898) Beitrag zur Ernahrungsphysiologie and zur Differential diagnose der Bakterien der hamorrhagischen Septicemia. Z. Hyg. 28, 20-32. 